\title{
Tofacitinib in the treatment of patients with rheumatoid arthritis: position statement of experts of the Polish Society for Rheumatology
}

\author{
Eugeniusz J. Kucharz ${ }^{1}$, Marcin Stajszczyk², Anna Kotulska-Kucharz ${ }^{1}$, Bogdan Batko ${ }^{3}$, \\ Marek Brzosko ${ }^{4}$, Sławomir Jeka ${ }^{5}$, Piotr Leszczyński ${ }^{6,7}$, Maria Majdan $^{8}$, Marzena Olesińska $^{9}$, \\ Włodzimierz Samborski ${ }^{10}$, Piotr Wiland ${ }^{11}$ \\ ${ }^{1}$ Department of Internal Medicine, Rheumatology, and Clinical Immunology, Medical University of Silesia in Katowice, Poland \\ ${ }^{2}$ Department of Rheumatology and Autoimmune Diseases, Silesian Centre for Rheumatology, Rehabilitation, and Disability \\ Prevention in Ustroń, Poland \\ ${ }^{3}$ Department of Rheumatology, J. Dietl Memorial Specialist Hospital in Kraków, Poland \\ ${ }^{4}$ Department of Rheumatology, Internal Medicine, and Geriatrics, Pomeranian Medical University in Szczecin, Poland \\ ${ }^{5}$ Department of Rheumatology and Systemic Connective Tissue Disorders, Jan Biziel University Hospital No. 2 in Bydgoszcz, \\ Ludwik Rydygier Collegium Medicum in Bydgoszcz, Nicolaus Copernicus University in Toruń, Poland \\ ${ }^{6}$ Department of Rheumatology and Rehabilitation, Poznań University of Medical Sciences, Poland \\ ${ }^{7}$ Department of Rheumatology and Osteoporosis, J. Struś Memorial Multidisciplinary Municipal Hospital in Poznań, Poland \\ ${ }^{8}$ Department of Rheumatology and Systemic Connective Tissue Disorders, Medical University of Lublin, Poland \\ ${ }^{9}$ Clinic of Connective Tissue Diseases, National Institute of Geriatrics, Rheumatology and Rehabilitation, Warsaw, Poland \\ ${ }^{10}$ Department of Rheumatology and Rehabilitation, Poznań University of Medical Sciences, Poland \\ ${ }^{11}$ Department of Rheumatology and Internal Medicine, Wrocław Medical University, Poland
}

\begin{abstract}
Tofacitinib is a newly approved small-molecule targeted synthetic disease-modifying antirheumatic drug. The drug was designed as a selective and specific inhibitor of pro-inflammatory receptor signalling. Tofacitinib inhibits the process of intracellular signalling from the receptor to the cellular nucleus and inhibits the inflammation process via a new pathway (inhibition of the Janus kinases), which is unavailable to biological medicines.

Tofacitinib has been approved for use in the treatment of patients with moderate to severe active RA. The drug may be used in combination with methotrexate or another conventional synthetic disease-modifying antirheumatic drug or in monotherapy. The efficacy of tofacitinib has been confirmed in several clinical trials. The drug inhibits radiographic progression of the disease. The innovative mechanism of action of tofacitinib is a noteworthy feature because it offers hope of effective treatment for patients who fail to respond to other drugs. The presented article discusses the mechanism of action and the clinical application of tofacitinib. Tofacitinib represents a new group of disease-modifying antirheumatic drugs that can be placed on an equal footing with biological drugs already available.
\end{abstract}

Key words: tofacitinib, rheumatoid arthritis, management.

\section{Introduction}

Rheumatoid arthritis (RA) is a chronic systemic inflammatory condition of autoimmune origin. The disease is characterised by synovitis, which results in complete joint damage and numerous extra-articular and systemic symptoms that jointly lead to disability and premature death. The cause of RA is unknown. The last two decades have seen a significant development in RA treatment modalities. This has been achieved by the introduction of

Address for correspondence:

Eugeniusz J. Kucharz, Department of Internal Medicine, Rheumatology and Clinical Immunology, Medical University of Silesia,

45/47 Ziołowa St., 40-635 Katowice, Poland; e-mail: ejkucharz@poczta.onet.pl

Submitted: 24.08.2018; Accepted: 29.08.2018 
biological medicines on the one hand and optimisation of general treatment principles and adoption of therapeutic strategies on the other. Patients with RA require a comprehensive approach to treatment, which, however, is based on pharmacotherapy [1]. The drugs with proven inhibitory effect on the process of structural joint damage are referred to as disease-modifying antirheumatic drugs (DMARDs). Disease-modifying antirheumatic drugs are divided into synthetic drugs and biological drugs [2]. Synthetic drugs are further divided into conventional synthetic drugs (csDMARDs) and targeted synthetic drugs (tsDMARDs). Biological medicines are divided into originators and biosimilars [3].

Due to unknown aetiology of RA, present-day treatment does not ensure a permanent cure. The aim of treatment is to achieve complete and sustained remission, which is the state in which joint inflammation is inhibited to such a degree that it does not lead to (or only to a minimal degree leads to) the development of further structural joint damage. Remission is determined, with a high level of probability, on the basis of disease activity assessment. Disease activity is measured using composite disease activity indices. Discussion of these indices is beyond the scope of this paper; it is available in handbooks. In patients who fail to achieve remission, low disease activity, as determined using the aforementioned composite disease activity indices, is a satisfactory treatment outcome.

According to the current strategy for the management of RA, failure to achieve lower disease activity after three months of treatment or remission (or low disease activity) after six months of treatment necessitates treatment adjustment following a step-wise strategy [4]. Even though an RA treatment target has been defined (treat to target, T2T), in clinical practice a high percentage of patients still fail to achieve the described and required reduction in disease activity. This is due to a number of reasons. It may be related to the patient's attitude (non-compliance, arbitrary dosage reduction, etc.), but it primarily results from primary or secondary inefficiency of treatment as well as its intolerance, which develops sooner or later [5, 6]. Biological medicines, being protein molecules, are characterised by immunogenicity, which may reduce treatment effectiveness [7]. Also, the course of disease may differ: some patients suffer from rapidly progressing "aggressive" inflammation, which poorly responds to treatment. According to several reports concerning rheumatology practice, about one fourth of patients fail to achieve remission or low disease activity. Some of the reports are even less optimistic. For instance, up to $70 \%$ of all patients with RA do not reach the desirable treatment target with conventional synthetic
DMARDs [8]. In nearly $60 \%$ of patients, methotrexate is discontinued because of adverse reactions, and in about $25 \%$ - because of laboratory abnormalities [9]. In 30-40\% of patients treated with biological medicines, treatment does not achieve the expected reduction in disease activity [5, 6, 10-13]. Given the above, in addition to treatment optimisation, attempts are being made to introduce new drugs with a different mechanism of action in the clinical practice, which should translate into an increased percentage of patients with long-term remission or low disease activity. Therefore, this article comprehensively discusses the use and properties of a drug representing the new DMARDs group. Its introduction will no doubt increase the capacity and effectiveness of RA treatment.

\section{Mechanism of action of disease- -modifying antirheumatic drugs}

Conventional synthetic drugs affect metabolic processes indirectly related to the activation of inflammation and immune system phenomena. Not all of these mechanisms are known. Methotrexate inhibits the inflammatory processes that leads to joint structural damage via the adenosine pathway, and to a lesser degree inhibits cell proliferation - it is a purine antagonist [14]. Leflunomide, and more precisely teriflunomide, its active metabolite, is a pyrimidine antagonist $[15,16]$. Thus, it inhibits the proliferation of activated lymphocytes, which require de novo synthesis of pyrimidines for cell division. Non-activated lymphocytes do not proliferate and do not need to synthesise pyrimidines. The mechanism of action of other conventional synthetic DMARDs is little known.

Biological medicines are "well-targeted" anti-inflammatory drugs. Pro-inflammatory cytokines play a significant role in the development of inflammation. They are proteins produced by specific cells and released into the extracellular environment. There, they reach the cells responsible for further development of inflammation, bind to the extracellular domain of the specific receptor, and initiate further activation of the inflammatory process. The inhibition of inflammation by biologics consists of the reduction of pro-inflammatory cytokine activity (e.g. by way of binding of a specific antibody to the cytokine or inactivating a cytokine-specific receptor on the target cell). Some biologics used in the treatment of patients with RA have a different mechanism of action (e.g. they block adhesive proteins or reduce the population of immunocompetent cells). Due to their structure, all biological medicines act in the extracellular environment. Because of protein structure, they may be administered only parenterally. 


\section{Tofacitinib as a drug with a different mechanism of action}

Tofacitinib is a small-molecule targeted synthetic disease-modifying antirheumatic drug. It was designed as a selective and specific inhibitor of pro-inflammatory receptor signalling. As was previously discussed, pro-inflammatory cytokines reach the effector cells and bind to the extracellular domain of specific receptors. This initiates the process of signal transduction from the receptors to the inside of the cell. The initiation of transcription and expression of certain genes within the cellular nucleus is the last stage of intracellular receptor signal transduction. This results in the de novo synthesis of certain proteins, which are responsible for activating inflammation, e.g. the production of cytokines or proteins that stimulate cellular proliferation or migration, activation of inflammatory angiogenesis, etc.

Tofacitinib inhibits the process of intracellular signalling from the receptor to the cellular nucleus [17]. Thus, it complements the capacity to inhibit the inflammation process via a new pathway that is unavailable to biological medicines.

Receptor signalling is a multi-step process. The binding of an agonist (usually a pro-inflammatory cytokine) to the extracellular receptor domain leads to the binding of two components of the receptor molecules. As a result, two molecules of Janus kinases bound to the intracellular receptor domain are activated. Janus kinases are a group of enzymes that exhibit tyrosine kinase activity. The enzymes phosphorylate tyrosine residues in protein polypeptide chains. Phosphorylation affects the conformation of proteins in such a way that they may bind to other proteins that have the $\mathrm{SH} 2$ domain. As a result of phosphorylation, signal transducer and activator of transcription (STAT) proteins bind to the intracellular receptor domain, forming homodimers or heterodimers and undergo further phosphorylation. Subsequently, dimers move to the cell nucleus and initiate the transcription and expression of certain genes necessary for further activation of inflammation [18].

Four Janus kinases are known: JAK1, JAK2, JAK3, and TYK2 (tyrosine kinase 2). Initially, the term "JAK" stood for "Just Another Kinase". However, since two opposing domains were discovered in the enzymes concerned (i.e. the kinase domain that catalyses phosphorylation and the domain that inhibits the process), the term "Janus kinases", after the Roman god Janus, has been used [19].

Tofacitinib inhibits the activity of Janus kinases. It is a relatively small molecule, which has been obtained through chemical synthesis. The molecular formula of tofacitinib is $\mathrm{C}_{16} \mathrm{H}_{20} \mathrm{~N}_{6} \mathrm{O}$; the structural one is presented in Figure 1.

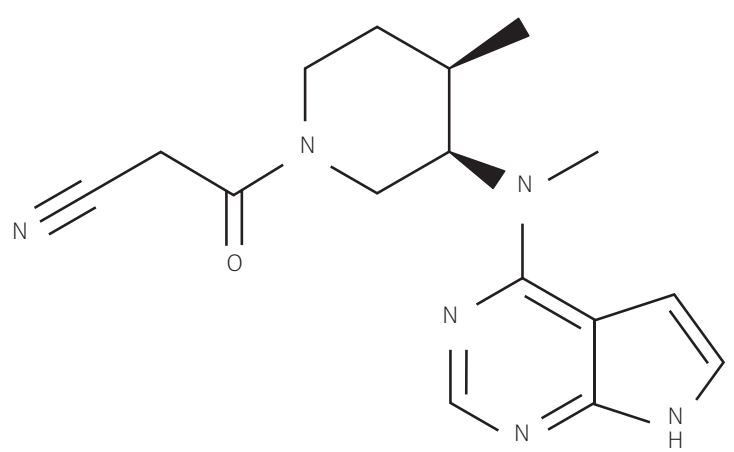

Fig. 1. Chemical structure of tofacitinib.

In the US, tofacitinib was approved by the US Food and Drug Administration in November 2012. In Europe, the European Medicines Agency approved tofacitinib in March 2017. Tofacitinib inhibits the activity of Janus kinases JAK1 and JAK3 and, to a lesser degree, JAK2 and TYK2. The inhibition of JAK1 and JAK3 by tofacitinib weakens the transmission of signals from interleukin (IL-2, IL-4, IL-6, IL-7, IL-9, IL-15, IL-21) and type I and II interferon receptors, which results in a change in immune and inflammatory responses. Other immune mechanism involved in pathogenesis of RA have been suggested [20]. Recently, impairment of monocyte-derived dendritic cell differentiation induced by tofacitinib in RA patients has been reported [21].

Tofacitinib significantly reduces inflammation in patients with RA. This effect is manifested as early (occurring as soon as in treatment week two) and significant reduction in serum C-reactive protein levels, maintained throughout the treatment period (a six-month treatment period was studied). It is sustained for at least two weeks after drug discontinuation [17].

Tofacitinib-treated patients with RA have a low circulating CD16/56+ NK cell count. It is most prominent in the second or third month of treatment. The change in the circulating NK cell count is reversed approximately two-to-six weeks after drug discontinuation [17].

In addition, the drug increases the B-cell count but has no impact on the T-cell count or subpopulations of these cells. Long-term use of tofacitinib (for approximately five years) has been demonstrated to decrease the CD4 and CD8 lymphocyte count by about one fourth. No relationship between these changes and infection rates has been found. Likewise, the use of tofacitinib has no effect on total serum immunoglobulin G, $M$, or A levels [17].

\section{Tofacitinib in the treatment of patients with rheumatoid arthritis - clinical trials}

Tofacitinib has been approved for use in the treatment of moderate-to-severe active RA in patients with inadequate response to, or intolerance of, one or more 
csDMARD(s) [17]. Tofacitinib has been studied in a comprehensive development programme in practically every patient population - from treatment-naïve patients (ORAL START), through non-responders, to methotrexate and other conventional synthetic disease-modifying antirheumatic drugs (ORAL SOLO, SYNC, SCAN, STANDARD, STRATEGY), non-responders, and biological medicines (ORAL STEP).

Tofacitinib may be used in combination with methotrexate or another cSDMARD or in monotherapy, which has been confirmed in clinical trials such as ORAL SOLO and ORAL SYNC [22, 23].

\section{Efficacy of monotherapy}

The efficacy of tofacitinib monotherapy has been demonstrated in the ORAL SOLO and START trials. The drug inhibits radiographic progression of the disease. The first effects (ACR20) are observed as soon as in the second week of treatment. In ORAL STRATEGY, tofacitinib in combination with methotrexate was demonstrated to be non-inferior to adalimumab in combination with methotrexate. The studies also confirm its beneficial effects on the patient's quality of life ORAL START was a clinical trial investigating the use of tofacitinib monotherapy in methotrexate-naïve patients. The study involved 958 subjects. After six months, ACR70 was achieved in $25.5 \%$ of subjects who received tofacitinib $5 \mathrm{mg}$ bid, $37.7 \%$ of subjects who received tofacitinib $10 \mathrm{mg}$ bid, and $12 \%$ of subjects in the methotrexate group. In addition, radiographic disease progression was assessed using modified Sharp/van der Heijde score. The study showed that tofacitinib monotherapy was significantly more effective in reducing disease activity and inhibiting radiographic progression than methotrexate [24]. It should be noted that currently the drug is not recommended in methotrexate-naïve patients or in patients in whom methotrexate is not contraindicated.

In ORAL SOLO, tofacitinib efficacy and safety were assessed in over 610 subjects with inadequate response to csDMARDs. Subjects were randomly assigned to receiving tofacitinib $5 \mathrm{mg}$ or $10 \mathrm{mg}$ bid or placebo for three months followed by tofacitinib $10 \mathrm{mg}$ for three more months. The primary endpoint at month 3 (ACR20) was achieved in $59.8 \%$ and $65.7 \%$ of subjects, respectively, vs. $26.7 \%$ of subjects in the placebo group $(p<0.001)$. DAS28-ESR < 2.6 was achieved in 5.6\%, 8.7\%, and $4.4 \%$ of subjects, respectively. The change in the quality of life as assessed using the HAQ-DI score was -0.50 and -0.57 vs. -0.19 ( $p<0.001)$. The most common AEs included headache and upper respiratory tract infection. The study confirmed that tofacitinib may be used as monotherapy after failure of cSDMARDs [25].

\section{Efficacy of combination treatment}

ORAL SYNC investigated the use of tofacitinib in patients with RA, who failed to respond or responded inadequately to DMARDs (methotrexate and other CSDMARD as well as biologics - TNF- $\alpha$ antagonists). The drug was used in combination with non-biological DMARDs. Over 792 subjects were randomly assigned to receive the study drug at the dose of $5 \mathrm{mg}$ or $10 \mathrm{mg}$ bid or placebo for three months followed by tofacitinib in two doses. ACR20 was achieved in over $50 \%$ of tofacitinib-treated subjects. The percentage of subjects with remission after six months was $8.5 \%$ and $12.5 \%$, respectively, vs. $2.6 \%$ in the placebo group. The study confirmed the efficacy of tofacitinib in combination with methotrexate or other csDMARDs in non-responders to DMARDs [26].

\section{Inhibition of structural damage progression}

ORAL SCAN was aimed at assessing the extent of inhibition of radiographic disease progression in subjects with inadequate response to methotrexate. Tofacitinib at the dose of $5 \mathrm{mg}$ and $10 \mathrm{mg}$ bid in combination with MTX was assessed. The paper has shown that tofacitinib inhibits progression of structural joint damage and decreases disease activity [27]. The endpoint of greater inhibition of radiographic progression in tofacitinib-treated subjects as compared with controls was not achieved. Minimal progression of structural damage was seen in both groups, which may have been related to long disease duration and initial advancement of radiographic changes.

\section{Efficacy after failure of conventional synthetic disease-modifying antirheumatic drugs}

The 12-month Phase III ORAL STANDARD study involved 717 subjects who received methotrexate. Subjects were randomly assigned to receiving tofacitinib $5 \mathrm{mg}$ or $10 \mathrm{mg}$ bid, adalimumab (40 mg every two weeks), or placebo. At month 3, subjects in the placebo group who failed to achieve a $20 \%$ reduction in the swollen or tender joint count were randomised to tofacitinib groups. At month 6, other subjects in the placebo group were randomly assigned to receive tofacitinib. The rates of ACR20 at month 6 were higher for tofacitinib $(5 \mathrm{mg}$ or $10 \mathrm{mg}$ ) and adalimumab (51.5\%, 52.6\%, and $47.2 \%$, respectively) vs. placebo (28.3\%). Also, improvements in the quality of life (HAQ-DI) and the percentage of subjects with disease activity DAS28-ESR $<2.6$ were greater in the active treatment group. To sum up, the study demonstrated that tofacitinib in combination with methotrexate is significantly more effective than placebo and has similar efficacy to adalimumab [28]. 
ORAL Strategy assessed efficacy of tofacitinib monotherapy at the dose of $5 \mathrm{mg}$ bid $(n=384)$ or in combination with methotrexate $(n=376)$ vs. adalimumab in combination with methotrexate $(n=386)$, in subjects with inadequate response to cSDMARDs. It was a 12-month double-blind Phase IIIb/IV study. After six months, ACR50 was achieved in $38 \%$ of subjects in the first group, $46 \%$ in the second group, and $44 \%$ in the adalimumab group. The paper demonstrated non-inferiority of tofacitinib in combination with methotrexate vs. adalimumab in combination with methotrexate [26].

\section{Efficacy after failure of biological treatment}

ORAL STEP was a six-month study to assess the efficacy of tofacitinib (5 mg or $10 \mathrm{mg}$ bid) in combination with methotrexate in the population of non-responders to biological treatment with TNF- $\alpha$ inhibitors vs. methotrexate alone. The study involved about $400 \mathrm{sub}$ jects. At month 3, ACR20 was achieved in $41.7 \%$ of subjects receiving tofacitinib $5 \mathrm{mg}$ bid (55/132) and 48\% (64/133) receiving tofacitinib at a higher dose. Among methotrexate-treated subjects, ACR20 was achieved in $24.4 \%(32 / 131)$. DAS28 < 2.6 was achieved in $6.7 \%$, $8.8 \%$, and $1.7 \%$ of subjects. The most common AEs included diarrhoea, headache, and nasopharyngitis (approximately $4 \%$ each). The paper confirmed that tofacitinib enabled achievement of early and good clinical response in non-responders to biological treatment with TNF- $\alpha$ inhibitors [28], although clinical remission in this population was achieved only in a small proportion of subjects after such a short treatment time. Registry data may help to provide an answer to the question of whether remission rates in the population of tofacitinib-treated patients may be higher with longer treatment duration.

\section{Sustained therapeutic effects}

Studies of long-term safety confirm the safety profile established in pivotal clinical trials as well as sustained efficacy of tofacitinib. In 2017, Cohen et al. [29] published an analysis involving 6194 tofacitinib-treated patients $(19,406$ patient-years; mean treatment duration was 3.4 years). They demonstrated that ACR20/50/70 rates remained stable and did not significantly differ between monotherapy and combination treatment over a nineyear follow-up. DAS28-CRP and CDAI were likewise sustained [30]. In February 2018, the results of another study were published, in which the use of tofacitinib in clinical practice was assessed over a period of three years [29]. The study involved 9291 patients (34,223 patient-years). No changes in the long-term safety profile or differences in drug safety when used in clinical practice vs. clinical trials were identified.

\section{The role of tofacitinib in current treatment strategies}

In 2016, an algorithm for the treatment of RA was updated by the expert force of the European League Against Rheumatism (EULAR), and in 2015 [2] by the American College of Rheumatology (ACR) [31]. The recommendations emphasise the role of early diagnosis of RA and early initiation of treatment with methotrexate, or leflunomide or sulfasalazine if methotrexate is contraindicated. Failure of the first phase of treatment, i.e. lack of decrease in disease activity after three months and lack of remission (or low disease activity) after six months, is an indication for entering the second phase of treatment strategy. A similar approach is required if the drugs used in the first phase are not tolerated. When starting the second phase of treatment, one should check if there are factors indicative of a rapid and "aggressive" course of disease. If such factors are involved, a biological medicine is started and methotrexate is usually continued, if tolerated.

The EULAR 2016 recommendations [4] offer an alternative that is considered as equivalent. The alternative option is the use of a Janus kinase inhibitor. It can be used as monotherapy or in combination with methotrexate. This phase of treatment, as all other phases, is aimed at achieving remission or low disease activity before a predetermined time point (six months). If the treatment target has not been reached, another biological drug or a Janus kinase inhibitor should be used.

One year earlier (in 2015), ACR treatment recommendations were published. They distinguish patients with early RA (i.e. symptoms lasting less than six months) from those with established RA (clinically active for at least six months). Janus kinase inhibitors are recommended for use after failure of conventional DMARD monotherapy in the treatment of established RA [32].

Since the publication of these recommendations, new tofacitinib studies have been reported, which even better document the drug use in the treatment strategy.

\section{Registry observations}

Tofacitinib data include information obtained in clinical trials as well as data gathered and analysed in a number of registries such as the American Corrona registry, a Swiss registry, the German RABBIT registry, and Turkish and Russian registries.

The American Corrona (Consortium of Rheumatology Researchers of North America) registry was established in 2000 by experienced clinical and academic 
rheumatologists. The registry collects data submitted by 670 rheumatologists from 170 centres across the US. By 30 June 2017, the Corrona registry recorded data concerning over 44,500 patients and over 152,000 patient-years. The available data show that the US population of tofacitinib-treated patients is growing, and its demographic profile is not significantly different from that of patients who are started on biological treatment. However, tofacitinib is more often used as monotherapy [33].

\section{Safety profile of tofacitinib}

The safety profile of tofacitinib was assessed in the aforementioned clinical trials as well as in the real-world setting. In accordance with the Summary of Product Characteristics, the most common adverse reactions reported during the first three months of treatment included headache, nausea, diarrhoea, upper respiratory tract infection, and nasopharyngitis.

Although the drug is new, we have data from nearly 10 years of clinical observation. The summary of data concerning eight years of tofacitinib use provides important information [29]. It emphasises that the safety profile of tofacitinib is similar to that of currently used biological medicines. During the ACR2017 congress, analyses of nearly 10 years of tofacitinib use were presented. Their results proved consistent with data already known from clinical trials [30]. What is important, the safety profile established in clinical trials was confirmed in clinical practice. Data from the Corrona registry (US) show that the risk of serious infections, tuberculosis, neoplasms, cardiovascular events, and gastrointestinal perforation is similar to that related to biological medicines. Also, publications from Japan and Latin America are consistent with the previously reported safety profile [34-36].

A paper by Tarp et al. [37] - a systematic review and a meta-analysis of data on biological and targeted medicines - is an interesting summary of the safety profile of tofacitinib. It demonstrated that tofacitinib monotherapy was associated with significantly lower rates of serious adverse events as compared with adalimumab, tocilizumab monotherapy, and tofacitinib in combination with cSDMARD. Overall, tofacitinib was not associated with a significantly increased risk of serious adverse events as compared to biological medicines.

Strand et al. [38] analysed the risk of serious infections in tofacitinib-treated patients. According to the results reported, the rate of serious infections was 2.53.02/100 patient-years for tofacitinib $5 \mathrm{mg}$ bid vs. up to 4.9/100 patient-years for TNF- $\alpha$ inhibitors.

Only in the case of herpes zoster infection was the rate slightly higher for tofacitinib; however, the ef- fect differed by geographical region - the highest risk was seen in Asian countries (Japan), while the lowest was in Central and Eastern Europe (IR 8 vs. 2.4/100 patient-years). Furthermore, the majority of cases were mild and uncomplicated (approx. 90\%); in over 90\% of cases only one dermatome was involved. Most subjects did not have to resign from tofacitinib treatment (only $8 \%$ of patients required complete treatment discontinuation, 40\% required treatment interruption, and 40\% continued treatment). It is worth noting that infection rates did not increase with time [39].

\section{Special situations}

It is recommended that prior to tofacitinib initiation all patients receive all mandatory vaccinations as per the current guidelines for vaccination in patients with inflammatory rheumatic diseases. In addition, it is emphasised that live vaccines should not be co-administered with tofacitinib. When deciding to use live vaccines before tofacitinib is started, the level of immunocompetence of a given patient should be taken into consideration. Live vaccines should be administered at least two and preferably four weeks before administration of tofacitinib is started [17]. Vaccination efficacy studies showed that response to influenza vaccination was similar to that in the placebo group (approximately 60\%). In the case of pneumococcal vaccines, the response rate was slightly lower among patients receiving tofacitinib plus methotrexate: $32 \%$ among patients receiving tofacitinib plus methotrexate, $62 \%$ among patients receiving tofacitinib monotherapy, $62 \%$ among patients receiving methotrexate monotherapy, and $77 \%$ among patients receiving placebo [40].

There are no adequate and well-controlled studies on the use of tofacitinib in pregnant women. Tofacitinib was shown to be teratogenic in rats and rabbits and have effects on parturition as well as perinatal and postnatal development. Tofacitinib is contraindicated during pregnancy. Women of childbearing potential should be advised to use effective contraception during treatment and for at least four weeks after the last dose. Tofacitinib is secreted in human milk. A risk to the breast-fed child cannot be excluded. Therefore, tofacitinib is contraindicated during breastfeeding [17].

What clearly distinguishes tofacitinib from other currently used drugs is a completely different mechanism of action. Tofacitinib, as an intracellular Janus kinase inhibitor, inhibits the inflammatory process and affects the expression of pro-inflammatory cytokines $[39,41]$. What distinguishes tofacitinib from biological medicines is the fact that it can be administered orally, which is the preferred route of administration for some 
patients, and reduces the burden on the healthcare system $[42,43]$. It is also worth noting that the drug can be crushed and administered with water, which is important for individuals with dysphagia.

Another important aspect is its short half-life (three hours), which plays a role in the event of elective procedures such as orthopaedic surgeries. The American College of Rheumatology recommends interruption of tofacitinib therapy seven days before an elective major surgery, which is significantly less than in the case of TNF- $\alpha$ inhibitors [44].

In accordance with the Summary of Product Characteristics, TNF- $\alpha$ inhibitors are contraindicated in individuals with NYHA class III/IV heart failure, while tofacitinib is not [17]. Tofacitinib can be used in individuals with severe renal impairment, also in those undergoing dialysis, although eGFR $<30 \mathrm{ml} / \mathrm{min} / 1.73 \mathrm{~m}^{2}$ necessitates a dose reduction to $5 \mathrm{mg}$ daily [17].

The drug may be safely used in individuals with mild hepatic impairment (Child-Pugh class A). Only individuals with severe impairment (class C) cannot use the drug [17]. Tofacitinib has a beneficial effect on pain, and an analysis of data from clinical trials shows that a significant alleviation of pain is achieved as soon as after two weeks and is maintained over time [45].

No dose adjustment is required in subjects with mild (creatinine clearance $50-80 \mathrm{ml} / \mathrm{min}$ ) or moderate (creatinine clearance 30-49 $\mathrm{ml} / \mathrm{min}$ ) renal impairment. Subjects with severe renal impairment (creatinine clearance $<30 \mathrm{ml} / \mathrm{min}$ ) require dose reduction to $5 \mathrm{mg}$ daily. No dose adjustment is necessary in patients aged 65 years or older, although data on patients aged 75 years and older are limited.

Immunogenicity, which may affect treatment outcomes, is not expected in the case of tofacitinib due to its chemical structure $[7,46]$. In accordance with the label (summary of product characteristics), tofacitinib may be used with methotrexate and in monotherapy. Favourable clinical outcomes of such treatment were demonstrated in the previously mentioned studies (ORAL SOLO, START).

During the ACR Meeting (San Diego 2017), a study was reported that demonstrated that the effects tofacitinib treatment are independent of body mass index, which distinguishes it from many other drugs [47]. The risk of thromboembolic events is not increased with tofacitinib [48].

\section{Interactions between tofacitinib and other drugs}

Tofacitinib is metabolised in the liver by CYP3A4 and, to a lesser degree, by CYP2C19. Therefore, interactions with other substances, drugs included, which are me- tabolised in the same way, are likely. Interactions with ketoconazole, fluconazole, tacrolimus, and ciclosporin A are possible - these medicines may increase tofacitinib concentration.

Co-administration with rifampicin (CYP inducer) may reduce tofacitinib concentration. This is particularly important in the case of individuals with latent tuberculosis in whom antimycobacterial treatment had been started before tofacitinib. If tofacitinib is started while rifampicin is used, reduced effectiveness of the standard dose should be taken into consideration. This does not apply to a combination treatment with isoniazid, which does not interact with tofacitinib. It should be underlined that methotrexate at therapeutic doses has been demonstrated to have no effect on tofacitinib pharmacokinetics. Likewise, tofacitinib has no effect on the pharmacokinetics of oral contraceptives or metformin [17].

\section{Conclusions}

The introduction of tofacitinib for the treatment of patients with RA opens an era of new therapeutic options. According to treatment strategies prepared on the basis of data published in 2014/2015, tofacitinib is recommended for use after failure or intolerance of conventional synthetic disease-modifying anti-rheumatic drug(s). The drug was approved for treating patients with RA, who failed to achieve response (inadequate effectiveness) or were intolerant of one or several conventional synthetic $\operatorname{DMARD}(\mathrm{s})$. It is recommended that it be used in combination with methotrexate, but it may also be used as monotherapy if methotrexate is not tolerated or is contraindicated.

Currently available study results fully confirm the rationale for the above recommendation and suggest that the approved indications may be extended in the future and the drug may prove useful in treating other diseases. The innovative mechanism of action of tofacitinib is a noteworthy feature because it offers hope for effective treatment for patients who fail to respond to other drugs. The route of administration also plays a role because it is an additional factor distinguishing the drug from biological medicines.

This work was prepared with financial support from Pfizer Polska Sp. z o.o., and the authors were receiving consulting fees for their expert opinions.

\section{References}

1. Kucharz EJ. Reumatoidalne zapalenie stawów. In: Puszczewicz MJ (ed.). Wielka Interna. Medical Tribune, Warszawa 2016: 79-95. 
2. Smolen JS, van der Heijde D, Machold KP, et al. Proposal for a new nomenclature of disease-modifying antirheumatic drugs. Ann Rheum Dis 2014; 73: 3-5.

3. Kucharz EJ, Stajszczyk M, Batko B, et al. Biopodobne leki biologiczne w reumatologii. Forum Reumatol 2017; 4: 191-204.

4. Smolen JS, Landewé R, Bijlsma J, et al. EULAR recommendations for the management of rheumatoid arthritis with synthetic and biological disease-modifying antirheumatic drugs: 2016 update. Ann Rheum Dis 2017; 76: 960-977.

5. Taylor PC, Sullivan E, Wood R, et al. Factors influencing treatment adjustments in rheumatoid arthritis patients - biologic DMARD treatment start and options. Arthritis Rheumato 2015; 67 (Suppl 1): abstract no 2640.

6. Rubbert-Roth A, Finckh A. Treatment options in patients with rheumatoid arthritis failing initial TNF inhibitor therapy: a critical review Arthritis Res Ther 2009; 11 (Suppl 1): S1.

7. Garcês S, Demengeot J, Benito-Garcia E. The immunogenicity of anti-TNF therapy in immune-mediated inflammatory diseases: a systematic review of the literature with a meta-analysis. Ann Rheum Dis 2013; 72: 1947-1955.

8. van Vollenhoven RF, Geborek P, Forslind K, et al. Conventional combination treatment versus biological treatment in methotrexate-refractory early rheumatoid arthritis: 2 year follow-up of the randomised, non-blinded, parallel-group Swefot trial. Lancet 2012; 379: 1712-1720.

9. Nikiphorou E, Negoescu A, Fitzpatrick JD, et al. Indispensable or intolerable? Methotrexate in patients with rheumatoid and psoriatic arthritis: a retrospective review of discontinuation rates from a large UK cohort. Clin Rheumatol 2014; 33: 609614.

10. Weinblatt ME, Keystone EC, Furst DE, et al. Adalimumab, a fully human anti-tumor necrosis factor alpha monoclonal antibody, for the treatment of rheumatoid arthritis in patients taking concomitant methotrexate: the ARMADA trial. Arthritis Rheum 2003; 48: 35-45.

11. Weinblatt ME, Kremer JM, Bankhurst $A D$, et al. A trial of etanercept, a recombinant tumor necrosis factor receptor: $\mathrm{Fc}$ fusion protein, in patients with rheumatoid arthritis receiving methotrexate. N Engl J Med 1999; 340: 253-259.

12. Lipsky PE, van der Heijde DM, St Clair EW, et al. Infliximab and methotrexate in the treatment of rheumatoid arthritis. Anti-Tumor Necrosis Factor Trial in Rheumatoid Arthritis with Concomitant Therapy Study Group. N Engl J Med 2000; 343: 1594-1602

13. St Clair EW, van der Heijde DM, Smolen JS, et al. Combination of infliximab and methotrexate therapy for early rheumatoid arthritis: a randomized, controlled trial Arthritis Rheum 2004; 50: 3432-3443.

14. Tian $\mathrm{H}$, Cronstein BN. Understanding the mechanisms of action of methotrexate: implications for the treatment of rheumatoid arthritis. Bull NYU Hosp Jt Dis 2007; 65: 168-173.

15. Kucharz EJ. Zastosowanie leflunomidu w leczeniu chorych na reumatoidalne zapalenie stawów. Pol Arch Med Wewn 2001; 105: 177-182.

16. Fragoso YD, Brooks JB. Leflunomide and teriflunomide: altering the metabolism of pyrimidines for the treatment of autoimmune diseases. Expert Rev Clin Pharmacol 2015; 8: 315-320.
17. Tofacitinib: Summary of Product Characteristics. http://www. ema.europa.eu/docs/en_GB/document_library/EPAR_-_Product_Information/human/004214/WC500224911.pdf

18. Jiang JK, Ghoreschi K, Deflorian F, et al. Examining the chirality, conformation and selective kinase inhibition of $3-((3 R, 4 R)$ 4-methyl-3-(methyl(7H-pyrrolo[2,3-d]pyrimidin-4-yl)amino) piperidin-1-yl)-3-oxopropanenitrile (CP-690,550). J Med Chem 2008; 51: 8012-8018.

19. Kucharz EJ. Medical eponyms of mythological origin. Acta Neophilologica 2017; 19: 20-42.

20. Bartosińska J, Zakrzewska E, Król A, et al. Differential expression of programmed death 1 (PD-1) on CD4+ and CD8+ T cells in rheumatoid arthritis and psoriatic arthritis. Pol Arch Intern Med 2017; 127: 815-822.

21. Marzioli V, Canavan M, Wade S, et al. Tofacitinib impairs monocyte-derived dendritic cell differentiation in rheumatoid arthritis and psoriatic arthritis. Annual Congress of the European League Against Rheumatism, Amsterdam 2018, Abstract AB0046. Ann Rheum Dis 2018; 77 (Suppl): A1223.

22. Lee EB, Fleischmann R, Hall S, et al. Tofacitinib versus methotrexate in rheumatoid arthritis. N Engl J Med 2014; 370: 23772386.

23. Fleischmann R, Kremer J, Cush J, et al. Placebo-controlled trial of tofacitinib monotherapy in rheumatoid arthritis. N Engl J Med 2012; 367: 495-507.

24. Kremer J, Li ZG, Hall S, et al. Tofacitinib in combination with nonbiologic disease-modifying antirheumatic drugs in patients with active rheumatoid arthritis: a randomized trial. Ann Intern Med 2013; 159: 253-261.

25. van der Heijde D, Tanaka Y, Fleischmann R, et al. Tofacitinib (CP-690,550) in patients with rheumatoid arthritis receiving methotrexate twelve-month data From a Twenty-Four-Month Phase III Randomized Radiographic Study. Arthritis Rheum 2013; 65: 559-570.

26. van Vollenhoven R, Fleischmann R, Cohen S, et al. Tofacitinib or adalimumab versus placebo in rheumatoid arthritis. N Engl J Med 2012; 367: 508-519.

27. Burmester GR, Blanco R, Charles-Schoeman CH, et al. Tofacitinib $(\mathrm{CP}-690,550)$ in combination with methotrexate in patients with active rheumatoid arthritis with an inadequate response to tumour necrosis factor inhibitors: a randomised phase 3 trial. Lancet 2013; 381: 451-460.

28. Fleischmann R, Mysler E, Hall S, et al. Efficacy and safety of tofacitinib monotherapy, tofacitinib with methotrexate, and adalimumab with methotrexatein patients with rheumatoid arthritis (ORAL Strategy): a phase 3b/4, double-blind, head-tohead, randomized controlled trial. Lancet 2017; 390: 457-468.

29. Cohen SB, Tanaka Y, Mariette X, et al. Long-term safety of tofacitinib for the treatment of rheumatoid arthritis up to 8.5 years: integrated analysis of data from the global clinical trials. Ann Rheum Dis 2017; 76: 1253-1262.

30. Wollenhaupt J, Silverfield J, Lee EB, et al. Tofacitinib, an oral Janus kinase inhibitor, in the treatment of rheumatoid arthritis: safety and efficacy in open-label, long-term extension studies over 9 Years. Arthritis Rheumatol 2017; 69 (Suppl 10). http:// acrabstracts.org/abstract/tofacitinib-an-oral-janus-kinase-inhibitor-in-thetreatment-of-rheumatoid-arthritis-safety-and-efficacy-in-open-label-long-term-extensionstudies-over-9-years/. 
31. Cohen S, Curtis JR, DeMasi R, et al. Worldwide, 3-year, post-marketing surveillance experience with tofacitinib in rheumatoid arthritis. Rheumatol Ther (in print) 2018 Feb 22. doi: 10.1007/s40744-018-0097-3.

32. Singh JA, Saag KG, Bridges SL Jr, et al. 2015 American College of Rheumatology Guideline for the Treatment of Rheumatoid Arthritis. Arthritis Care Res 2016; 68: 1-25.

33. Harnett J, Gerber R, Gruben D, et al. Evaluation of Real-World Experience with Tofacitinib Compared with Adalimumab, Etanercept, and Abatacept in RA Patients with 1 Previous Biologic DMARD: Data from a U.S. Administrative Claims Database. J Manag Care Spec Pharm 2016; 22: 1457-1471.

34. Iwamoto N, Tsuji S, Takatani A, et al. Efficacy and safety at 24 weeks of daily clinical use of tofacitinib in patients with rheumatoid arthritis. PLoS ONE 2017; 12: e0177057.

35. Schneeberger EE, Salas A, Medina LF, et al. Real-world use of tofacitinib in rheumatoid arthritis: data from Latin America Ann Rheum Dis 2017; 76 (Suppl 2): 1196. http://ard.bmj.com/ content/annrheumdis/76/Suppl_2/1196.3.full.pdf

36. Mimori T, Harigai M, Atsumi T, et al. Post-marketing surveillance of tofacitinib in Japanese patients with rheumatoid arthritis: an interim report of safety data [abstract]. Arthritis Rheumatol 2017; 69 (Suppl 10). http://acrabstracts.org/ abstract/post-marketing-surveillance-of-tofacitinib-in-japanese-patients-with-rheumatoid-arthritis-an-interim-report-of-safety-data/ (accessed: 21.12.2017).

37. Tarp S, Furst DE, Boers M, et al. Risk of serious adverse effects of biological and targeted drugs in patients with rheumatoid arthritis: a systematic review meta-analysis. Rheumatology 2017; 56: 417-425.

38. Strand V, Ahadieh S, French J, et al. Systematic review and meta-analysis of serious infections with tofacitinib and biologic disease-modifying antirheumatic drug treatment in rheumatoid arthritis clinical trials. Arthritis Res Ther 2015; 17: 362-367.

39. Winthrop KL, Curtis JR, Lindsey S, et al. Herpes zoster and tofacitinib: Clinical outcomes and the risk of concomitant therapy. Arthritis Rheumatol 2017; 69: 1960-1968.

40. Winthrop KL, Silverfield J, Racewicz A, et al. The effect of tofacitinib on pneumococcal and influenza vaccine responses in rheumatoid arthritis. Ann Rheum Dis 2016; 75: 687-695.
41. Fleischmann R. Tofacitinib in the treatment of active rheumatoid arthritis in adults. Immunotherapy 2018; 10: 39-56.

42. Alten R, Krüger K, Rellecke J, et al. Examining patient preferences in the treatment of rheumatoid arthritis using a discrete-choice approach. Patient Prefer Adherence 2016; 10: 2217-2228.

43. Louder AM, Singh A, Saverno K, et al. Patient preferences regarding rheumatoid arthritis therapies: a conjoint analysis. Am Health Drug Benefits 2016; 9: 84-93.

44. Goodman SM, Springer B, Guyatt G, et al. 2017 American College of Rheumatology/American Association of Hip and Knee Surgeons Guideline for the Perioperative Management of Antirheumatic Medication in Patients With Rheumatic Diseases Undergoing Elective Total Hip or Total Knee Arthroplasty. Arthritis Rheumatol 2017; 69: 1538-1551.

45. Ogdie A, de Vlam K, Mclnnes IB, et al. Effect of Tofacitinib on reducing pain in patients with rheumatoid arthritis, psoriatic arthritis and ankylosing spondylitis [abstract]. Arthritis Rheumatol 2017; 69 (Suppl 10).

46. Bartelds GM, Krieckaert ChLM, Nurmohamed MT, et al. Development of antidrug antibodies against adalimumab and association with disease activity and treatment failure during long-term follow-up. JAMA 2011; 305: 1460-1468.

47. Dikranian A, Gonzalez-Gay MA, Wellborne F, et al. The efficacy of tofacitinib in patients with rheumatoid arthritis stratified by baseline Body Mass Index. Arthritis Rheumatol 2017; 69 (Suppl 10). http://acrabstracts.org/abstract/the-efficacy-oftofacitinib-in-patients-with-rheumatoid-arthritis-stratifiedby-baseline-body-mass-index (accessed: 20.12.2017).

48. Mease PJ, Kremer J, Cohen S, et al. Incidence of thromboembolic events in the management with tofacitinib patients with rheumatoid arthritis, psoriasis, psoriatic arthritis and ulcerative colitis development programs. Arthritis Rheumatol 2017; 69 (Suppl 10). http://acrabstracts.org/abstract/incidence-of-thromboembolic-events-in-the-tofacitinib-rheumatoid-arthritis-psoriasis-psoriatic-arthritis-and-ulcerative-colitis-development-programs/ 